SHIFT-AND-ADD-TYPE ALGORITHMS AND THEIR APPLICATION TO CAPELLA

William G. Bagnuolo, Jr.

The Aerospace Corp., Los Angeles, CA y0009

\title{
INTRODUCTION
}

Bates and Cady (1980) proposed a method for astronomical image reconstruction that involves superimposing regions about the brightest pixels of each frame produced by speckle interferometric techniques. Unlike the method of Lynds, Worden, and Harvey (1976), no attempt is made to center apparent speckle images before superposition. This method is hereafter referred to as "shift-and-add" (SAA). The simplicity of this method makes it highly suitable for analysis and simulation.

This paper summarizes the application of extensions of SAA to the problem of determining reliable double star intensity ratios. Theoretical and simulation results presented in a previous paper (hereafter called "paper I") by Bagnuolo (1982) will be summarized. In addition, the results of the application of SAAtype algorithms to Capella will be presented.

\section{THEORETICAL RESULTS}

Because of the limited number of frames used in speckle interferometry and noise considerations, the original SAA algorithm was extended by considering the superposition about pixels of any brightness leve1, not just the brightest pixels of a frame. The composite image resulting from superposing about pixels with $\mathrm{k}$ photons can be denoted by SAA(k). It can be shown that the autocorrelation (A) is just a weighted sum, i.e. $A=\sum n \operatorname{SAA}(n)$ :

Both the autocorrelation and SAA produce three "spots" for double stars, the "center", "primary", and "ghost" spots. The outer two spots for the autocorrelation are equal in intensity, but for SAA(n) for high $n$ the primary spot is brighter than the ghost spot (thereby resolving the usual $180^{\circ}$ position ambiguity). In principle, one could define a function $f(n) \neq n$ and create a composite image $B=\Sigma f(n)$ SAA(n) so that the true double star intensity ratio "f" could be determined optimally from the intensity ratio of the two outer spots, " $r$ ". In practice, it was found that for bright double stars the brightest or dimmest pixels could be used for superposition (i.e. $f(n)=1$ for $n$ sufficiently large or small, $f(n)=U$ otherwise).

The main results from the theoretical calculations of paper I were:

1) For bright double stars superposition about the top $1-2 \%$ or bottom $8 \%$ of the pixels produces an approximately linear relation between $r$ and $f$.

2) The SAA algorithm is better than the autocorrelation for determining magnitude differences for $\Delta \mathrm{m} \propto 1 \mathrm{mag}$. SAA also appears to be better than the autocorrelation for detecting dim components $(\Delta \mathrm{m}>3 \mathrm{mag}$.$) .$ 
3) SAA can resolve the $180^{\circ}$ position ambiguity for stars as dim as 16th magnitude for $l^{\prime \prime}$ seeing with a 5-m. telescope. Roughly two orders of magnitude more photons are required to resolve the position ambiguity than to detect the duplicity of a double star. This magnitude limit depends strongly upon the seeing disk size and rather weakly upon the telescope aperture.

2. APPLICATION OF SAA METHODS TO CAPELLA.

a) SAA algorithm results- A practical test of the SAA and related algorithms was furnished by Capella data obtained by $\mathrm{K}$. Hege (1981). Three hundred frames of data were taken with the 4-m KPNO telescope and camera. described elsewhere (Hege, 1983 ) at 5500 50\%. Each digitized frame consisted of $128 \times 128$ 8-bit pixels with a plate scale of 0:00705/pixel.

The data was analyzed by the following procedure. In each frame a 52-pixel wide, octagonal region was used for the SAA superpositions. Each region was centered on the intensity centroid of the frame. The frames were rectified for the overall Gaussian fall-off in intensity with distance from the center. This slowly-varying function was estimated by adding all the centered frames into a single "long-exposure", giving $\sigma=46.0$ pixels. (Runs without rectification gave basically the same results as below.)

The shift-and-add superpositions were done for the top $1 \%$ and bottom $8 \%$ of the pixels (in intensity) in each octagonal area. The $19 \times 19$ pixel composite images resulting from SAA were further analyzed by a 14-parameter least-squares fit which included the following parameters:

i) overall "tilt" of the form: $a_{1} x+a_{2} y+a_{3} x y$

ii) $(x, y)$ position of the primary spot

iii) heights and widths of the primary, ghost, and center spots, assuming a Gaussian shape. (Runs were also done by assuming that the primary and ghost spot widths were the same.) Because of the possibility of a small noise spike, the central 5 pixels were not fitted.

iv) the background, assuming a Gaussian plus constant model ( 3 parameters).

Figure 1 shows the primary and secondary spots after the fitted center and background spots have been removed. The intensity ratios of these spots gave $r=0.848$ for the top $1 \%$ and $r=0.836$ for the bottom $8 \%$ superpositions, respectively. The nominal errors were 0.018 and 0.020 respectively.

In addition to the calculations and simulations described in paper $I$, a number of new simulations were done to better relate $r$ to $f$. The procedure, suggested by J. C. Dainty (1982), involved putting a number of "patches" of random phase into an "aperture plane" modeled after the KPNO 4-m telescope's 
central obscuration. The resultant speckle pattern was convolved with a double star with the approximate separation (in pixels) of the actual data. According to the results of the models, $f=0.85$ and $f=0.81$ for the top $1 \%$ and bottom $8 \%$ respectively, giving a composite estimate of 0.83 for the intensity ratio.

The separation and position angle given by the parameter-fitting procedure described above was $P=0: 0420 \pm 0.0015$, and $\theta=333.0^{\circ} \pm 1.2^{\circ}$. This result compares to a value of $0: 0405$ and $145.8^{\circ}$ from McAlister's (1981) orbit for the observation date, 1981.092. The agreement in $\rho$ is good, but there is almost an $180^{\circ}$ difference in $\theta$. Bagnuolo and McAlister (1983) have analyzed a number of Capella frames whose absolute orientation was known with a similar result. (The value of the ascending node should therefore be ammended to $\Omega=220.2^{\circ}$.)

b) Screened Shift-and-Add Results- The original idea incorporated in SAA is that the regions about the brightest pixels would tend to form distorted replicas of the actual image. This idea can be extended by also requiring that the background about the bright pixels be minimal. This procedure, hereafter referred to as "screened shift-and-add" (SSAA), is a quantative version of what one does "instinctively" in looking for isolated speckle pairs on a double star speckle frame.

The main steps in the SSAA procedure are as follows:

i) The separation of the components is known already by means of the autocorrelation, $\mathrm{SAA}$, etc. The intensities in the central region of a frame are sampled at points given by the separation of the components. (Linear interpolation is used to get intensity values at non-integral pixel positions.) The sampling results in "strips" of data being produced.

For the Capella data, 33 strips 11 points long were produced for each frame. the distance between points was taken from the SAA results above. The separation between points in adjacent strips was about 2 pixels.

ii) Each data strip was examined for patterns of four adjacent points of the form $I_{1}-x-h-I_{2}$, where $h \geq a \bar{I}$ and $l_{1}$ and $I_{2} \leqslant h / b$, where $\bar{I}$ is the average intensity and $x$ is an unrestricted intensity. Parameters " $a$ " and " $b$ " are set low enough to generate a significant number of isolated bright speckles. The algorithm appears to be insensitive to the exact values of these coefficients. For $f=0.8$, for example, values of a between 1.5 and 3.0 , and $b$ between 2.5 and 4.0 yield intensity ratia estimates within 0.03 of each other. 
iii) For any occurrance of points satisfying the conditions of $i i$ ) the intensity ratio may be estimated by the background-subtracted ratio of $x$ to $h$, i.e. $r=\left(x-.5\left(1_{1}+l_{2}\right)\right) /\left(h-.5\left(1_{1}+l_{2}\right)\right)$. Figure 2 is a histogram of the fractional intensity, defined by $q=r /(1+r)$, for the Capella data.

Both the histogram and the composite four point image produced by summing all occurrances yield an intensity ratio estimate of $f=0.81 \pm .02$, in good agreement with the result obtained by SAA. Therefore, the best combined estimate of the intensity ratio appears to be $0.82 \pm .02$, which may be compared to a value of 0.794 ( $\Delta m=0.25$ ) found by Wright (1954), also at $5500 \AA$, by an analysis of the composite spectrum.

\section{CONCLUSION}

SAA-type algorithms appear to be a useful method of accurately determining double star intensity ratios. Such methods applied to multi-color data may independently determine the spectral types of the stars.

\section{REFERENCES:}

Bagnuolo, W.G., 1982, M.N.R.A.S. 200, 1113.

Bagnuolo, W.G., and McAlister, H.A., 1983, submitted to P.A.S.P. Bates, R.H.T., and Cady, F.M., 1980, Opt. Comm. 22, 365. Dainty, J.C., 1982, private communication.

Hege, E.K., I.A.U. Coll. \#62 Lynds, C.R., Worden, S.P., and Harvey, J.W., 1976. Ap.J. 207, 174. Wright, K.0., 1954, Ap.J. 119, 471. 


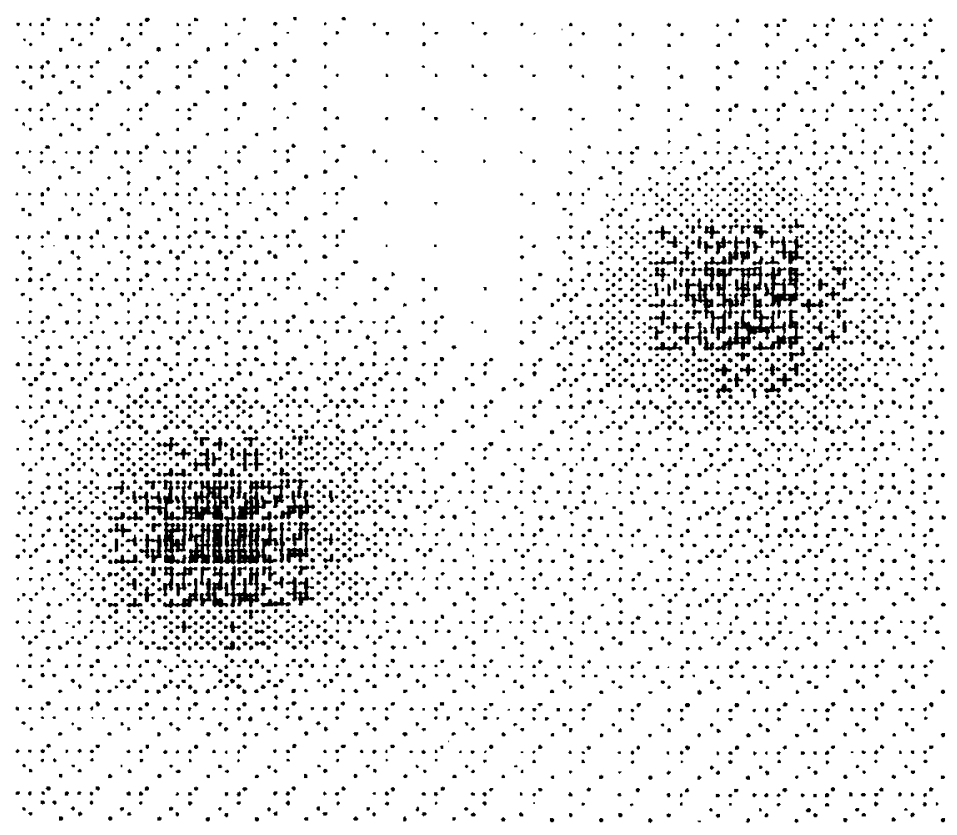

Figure 1. Primary, Ghost Spots for Capella, Top 1\% SAA A1gorithm

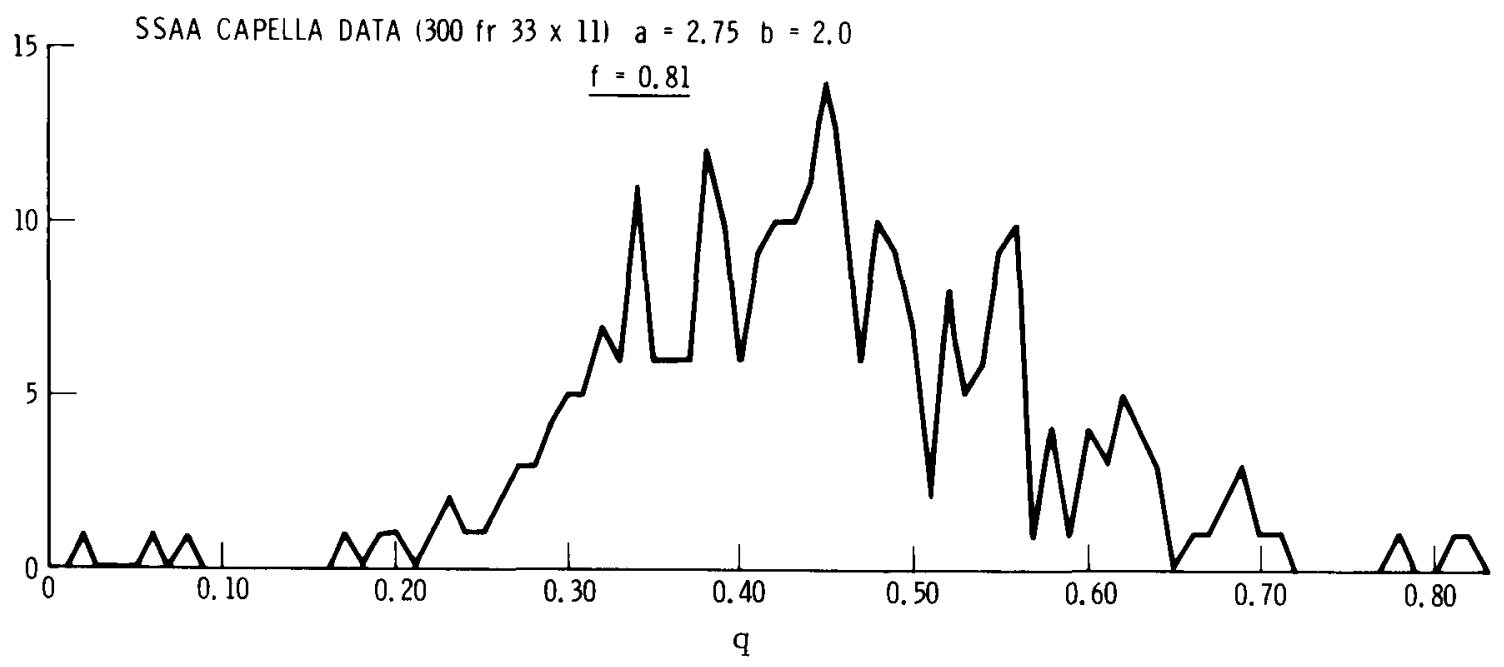

Figure 2. Capella, SSAA Algorithm 